Supporting Information

Quantifying the role of water in protein-carbohydrate interactions.

Sarah M. Tschampel and Robert J. Woods

\begin{tabular}{|c|c|c|c|c|c|c|c|c|c|c|c|}
\hline NAME & AA-H2O-HOR & & AA-H2O & & & & HOR & & & & $\begin{array}{l}\text { BSSE } \\
3 \text { body }\end{array}$ \\
\hline 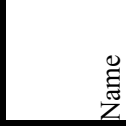 & 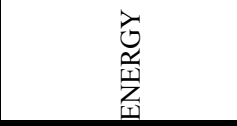 & in & 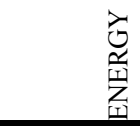 & 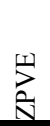 & $\begin{array}{l}\sqrt[w]{w} \\
w \\
0\end{array}$ & 武 & 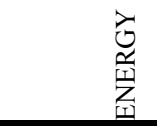 & $\stackrel{\text { In }}{\stackrel{1}{N}}$ & 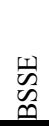 & 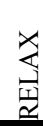 & لرّ \\
\hline
\end{tabular}

\title{
Threonine clusters
}

$\mathrm{TCRa}(\mathrm{R}=\mathrm{H}) \quad-308.059153 \quad 0.130611$

$\mathrm{TCRa}(\mathrm{R}=\mathrm{CH} 3 \quad-347.369102 \quad 0.159587$

$\mathrm{TCRb}(\mathrm{R}=\mathrm{H}) \quad-308.059153 \quad 0.130611$

$\mathrm{TCRb}\left(\mathrm{R}^{\prime}=\mathrm{CH}: \quad-347.369029 \quad 0.159631\right.$

$\mathrm{TL}(\mathrm{R}=\mathrm{H}) \quad-308.055461 \quad 0.129754$

$\mathrm{TL}(\mathrm{R}=\mathrm{CH} 3) \quad-347.365001 \quad 0.158672$

\section{Tyrosine clusters} $\mathrm{YCRa}\left(\mathrm{R}=\mathrm{CH}_{2} 2 \quad-539.174669 \quad 0.211406\right.$

$\mathrm{YCRb}(\mathrm{R}=\mathrm{H}) \quad-499.864594 \quad 0.182266$

$\mathrm{YCRb}(\mathrm{R}=\mathrm{CH}: \quad-539.174657 \quad 0.211089$

YLR $(\mathrm{R}=\mathrm{H}) \quad-499.857603 \quad 0.181063$

YLR $\left(\mathrm{R}=\mathrm{CH} 3^{\prime} \quad-539.167162 \quad 0.210153\right.$

\section{Lysine clusters}

$\mathrm{KBR}(\mathrm{R}=\mathrm{H}) \quad-249.237748$

$\mathrm{KBR}(\mathrm{R}=\mathrm{CH} 3) \quad-288.549756$

$\operatorname{KLR}(\mathrm{R}=\mathrm{H}) \quad-249.235109$

$\mathrm{KLR}(\mathrm{R}=\mathrm{CH} 3) \quad-288.546821$

0.127398

0.156795

0.128081

0.157508

\section{Arginine clusters}

\section{RLR $(\mathrm{R}=\mathrm{H}) \quad-398.148638$}

RLR (R-Cl

$\mathrm{RCRa}(\mathrm{R}=\mathrm{H}) \quad-398.145732$

$\mathrm{RCRa}(\mathrm{R}=\mathrm{CH} 3 \quad-437.457414$

$\mathrm{RCRb}(\mathrm{R}=\mathrm{H}) \quad-398.145732$

$\mathrm{RCRb}(\mathrm{R}=\mathrm{CH} 3$

$-437.456554$

\section{Aspartate clusters}

$\mathrm{DCR}(\mathrm{R}=\mathrm{H})$

$-381.599554 \quad 0.099004$

$-305.110853$

$-305.110853$

0.073409

0.073409

$-305.108175$

$-305.108236$

$-305.107820$

$-305.107782$

\section{Asparagine clusters}

$\begin{array}{lrr}\mathrm{NL} ' \mathrm{R}(\mathrm{R}=\mathrm{H}) & -362.249682 & 0.122273 \\ \mathrm{NL} \cdot \mathrm{R}(\mathrm{R}=\mathrm{CH} 3, & -401.560236 & 0.151755 \\ \mathrm{NCRa}(\mathrm{R}=\mathrm{H}) & -362.263666 & 0.124812 \\ \mathrm{NCRa}(\mathrm{R}=\mathrm{CH} 3 & -401.573377 & 0.153616 \\ \mathrm{NCRb}(\mathrm{R}=\mathrm{H}) & -362.263666 & 0.124812 \\ \mathrm{NCRb}(\mathrm{R}=\mathrm{CH}= & -401.573635 & 0.153726 \\ \mathrm{NLR}(\mathrm{R}=\mathrm{H}) & -362.252544 & 0.122859 \\ \mathrm{NLR}(\mathrm{R}=\mathrm{CH} 3) & -401.562891 & 0.152496\end{array}$

$-285.774871$ $-285.77487$ $-285.778791$ $-285.77879$ $-285.77487$ $-285.774871$ $-285.782341$

$-285.782341$

0.097037 0.097037 0.098003 0.098003 0.097037 0.097037 0.098937 0.098937
$-285.774392$ $-285.774394$ $-285.778024$ $-285.778053$

$-285.774530$ $-285.774562$ $-285.781611$ $-285.781575$
$-285.774276$ $-285.774208$ $-285.77766$ $-285.777652$ $-285.774236$ $-285.774205$ $-285.781493$ $-285.781383$

\section{$-76.464387$ $-115.774180$ \\ $-76.464387$ \\ $-115.774180$ \\ 0.021097 0.051293 0.021097 0.051293}

$-76.464631$ $-115.774066$ $-76.464658$ $-115.774132$
$-76.463881$ $-115.773765$ $-76.463738$ $-115.773631$ $-76.463994$ $-115.773825$
$-152.934527$ $-192.244164$ $-152.934527$ $-192.244813$ $-152.935850$ $-192.245375$

\section{Water}

( $\mathrm{R}=\mathrm{H})$

$\mathrm{b}(\mathrm{R}=\mathrm{H})$

c $(\mathrm{R}=\mathrm{H})$

a $(\mathrm{R}=\mathrm{CH} 3)$

$\mathrm{b}(\mathrm{R}=\mathrm{CH} 3)$

c $(\mathrm{R}=\mathrm{CH} 3)$
$-229.416865$ $-229.416865$ $-229.416865$ $-268.726815$ $-268.726808$

$-268.726892$
0.073168 0.073168 0.073168 0.102098 0.102182 0.102144
$-152.936447$ $-152.936447$ $-152.936447$ $-152.936447$ $-152.936447$ $-152.936447$
0.046191 0.046191 0.046191 0.046191 0.046191 0.046191
$-152.934530$ $-152.935269$ $-152.935247$

$-152.934514$ $-152.935240$ $-152.935223$
$-76.464387$ $-115.774180$ $-76.464387$ $-115.774180$

$-76.464387$

$-115.774180$
0.021097 0.051293 0.021097 0.051293 0.021097 0.051293
$-76.464644$ $-115.774192$

$-76.464857$ $-115.774196$

$-76.464379$ $-115.774148$
$-76.464026$ $-115.773860$

$-76.463852$ $-115.773712$ $-76.464016$ $-115.773856$
$-152.935447$ $-192.245705$ $-152.935447$ $-192.244934$ $-152.936244$ $-192.245740$ $\begin{array}{ll}-76.464387 & 0.021097\end{array}$ $\begin{array}{ll}-115.774180 & 0.051293\end{array}$
$-76.463203$ $-115.772560$
$-76.464290$ $-115.773742$ $-76.464292$ $-115.773810$
$-152.928126$ $-192.237673$ $-152.934386$ $-192.244451$

$-76.464302$ $-115.77388$ $-76.46427$ $-115.773672$

$-76.463952$ $-115.773736$
$-321.648106$ $-360.957669$ $-321.660118$ $-360.971210$ $-321.657355$ $-360.969250$ $\begin{array}{rr}-76.464387 & 0.021097 \\ -115.774180 & 0.051293 \\ -76.464387 & 0.021097 \\ -115.774180 & 0.051293 \\ -76.464387 & 0.021097 \\ -115.774180 & 0.051293 \\ -76.464387 & 0.021097 \\ -115.774180 & 0.051293\end{array}$
$-76.464729$ $-115.774374$

$-76.46416$ $-115.773957$

$-76.464155$ $-115.773955$ $-76.464717$ $-115.774405$
$-76.462666$ $-115.772106$
$-305.107063$ $-344.418176$

$$
\begin{array}{rr}
-76.464341 & -285.769230 \\
-115.774041 & -325.078810 \\
-76.463635 & -285.774530 \\
-115.773512 & -325.084681 \\
-76.463589 & -285.778024 \\
-115.773475 & -325.087575 \\
-76.464351 & -285.766842 \\
-115.774104 & -325.076584
\end{array}
$$

$-152.934169$ $-152.934898$ $-152.934907$ $-152.934099$ $-152.934821$ 
Supporting Information

Quantifying the role of water in protein-carbohydrate interactions.

Sarah M. Tschampel and Robert J. Woods

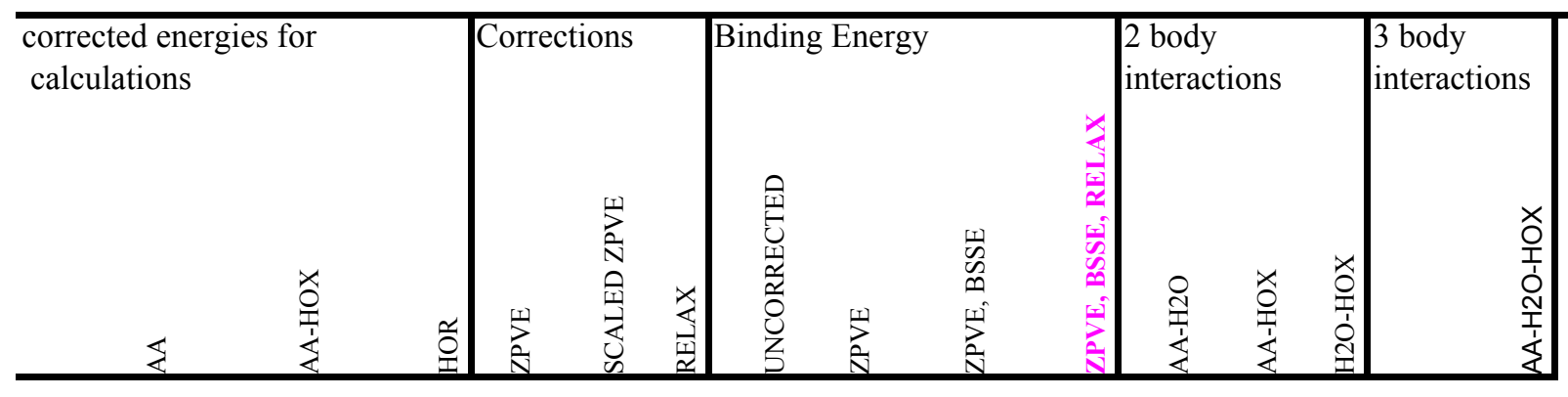

$\begin{array}{rr}-155.106544 & -231.576977 \\ -155.106498 & -270.887293 \\ -155.106544 & -231.578194 \\ -155.106575 & -270.887705 \\ -155.106573 & -231.572513 \\ -155.106603 & -270.882374\end{array}$

$\begin{array}{rrrrrr}-76.464192 & 3.14 & 3.07 & 2.15 & -9.54 & -6.46 \\ -76.464240 & 2.38 & 2.32 & 1.39 & -9.63 & -7.31 \\ -76.464337 & 3.41 & 3.34 & 0.54 & -10.47 & -7.14 \\ -76.464304 & 2.67 & 2.61 & 1.26 & -10.53 & -7.91 \\ -76.464421 & 2.60 & 2.55 & 0.60 & -7.22 & -4.67 \\ -76.464440 & 1.80 & 1.76 & 0.56 & -7.06 & -5.30\end{array}$

\section{$\begin{array}{lllll}-7.36 & -5.21 & -4.68 & -3.82 & -3.76\end{array}$}

$\begin{array}{lllll}-7.36 & -5.21 & -4.68 & -3.82 & -3.76 \\ -8.20 & -6.81 & -4.66 & -4.16 & -3.61\end{array}$

$-7.95$

$-8.70$

$-4.89$

$\begin{array}{llll}-7.41 & -3.82 & -4.68 & -3.76\end{array}$

$\begin{array}{llll}-7.43 & -3.83 & -4.46 & -4.07\end{array}$

$-5.48$

$\begin{array}{llll}-4.28 & -5.22 & -1.03 & -4.48 \\ -4.92 & -5.23 & -1.06 & -4.30\end{array}$

$-346.911617$

$\begin{array}{ll}-346.911657 & -462.690756 \\ -346.911617 & -423.384086\end{array}$

$-346.911503$

$-346.911784$

$-346.911812$ $-462.694628$

$\begin{array}{ll}-76.464385 & 3.12 \\ -76.464330 & 2.46\end{array}$

$\begin{array}{llllll}-76.464330 & 2.46 & 2.41 & 1.48 & -9.23 & -6.82\end{array}$

$\begin{array}{rrrrrrr}-423.377637 & -76.464695 & 2.77 & 2.70 & 0.57 & -6.81 & -4.11\end{array}$

$-462.687563$

$\begin{array}{rr}-76.464720 & 2.07\end{array}$

$\begin{array}{llll}2.03 & 0.52 & -6.67 & -4.64\end{array}$

$-6.99$

$\begin{array}{lllll}-7.86 & -6.38 & -5.06 & -3.04 & -4.47\end{array}$

$\begin{array}{lllll}-8.49 & -7.22 & -3.19 & -5.07 & -4.12\end{array}$

$\begin{array}{lllll}-9.55 & -8.25 & -3.20 & -5.62 & -3.92\end{array}$

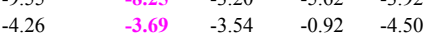

$\begin{array}{lllll}-4.76 & -4.24 & -3.55 & -0.99 & -4.30\end{array}$

\section{$-10.24$}

$-11.92$

$-12.72$

$-15.25$

$-11.29$

$-12.55$ $\begin{array}{rrrr}-9.64 & -15.24 & -4.68 & -3.76 \\ -11.00 & -15.25 & -4.74 & -4.31 \\ -7.53 & -10.52 & -11.95 & -2.99 \\ -9.36 & -10.20 & -13.10 & -3.53 \\ -7.51 & -11.95 & -10.52 & -2.99 \\ -8.75 & -12.05 & -11.97 & -2.74\end{array}$

$-14.92$

$\begin{array}{llll}-11.94 & -16.91 & -16.59 & -2.31\end{array}$

$\begin{array}{llll}-13.42 & -16.90 & -17.69 & -2.63\end{array}$

$-3.71$

$-4.41$

0.18
-0.01

-0.01
0.18
0.73 0.73

$-4.09$

$-5.10$

$-9.97$

$-10.76$

$-11.58$

$-12.45$

$-2.17$

$-2.95$

$\begin{array}{rrrr}-3.69 & -4.21 & -0.80 & -4.58 \\ -4.59 & -4.23 & -0.75 & -5.04 \\ -8.80 & -7.28 & -5.08 & -4.09 \\ -9.62 & -7.30 & -5.32 & -3.90 \\ -10.68 & -5.08 & -7.28 & -4.09 \\ -11.59 & -5.11 & -7.08 & -4.54 \\ -1.61 & -9.05 & 0.63 & -4.67 \\ -2.30 & -9.02 & 0.59 & -5.06\end{array}$

$-7.70$

$-7.29$

$-7.25$

$-8.58$

$-8.09$ $\begin{array}{llll}-5.95 & -3.75 & -4.20 & -4.17\end{array}$

$\begin{array}{llll}-5.97 & -4.17 & -3.75 & -4.20\end{array}$

$\begin{array}{llll}-5.97 & -4.20 & -4.17 & -3.75\end{array}$

$\begin{array}{llll}-6.83 & -3.71 & -3.99 & -4.05\end{array}$

$\begin{array}{llll}-6.78 & -4.16 & -4.01 & -4.07 \\ -6.86 & -4.19 & -4.54 & -3.60\end{array}$ 